\title{
Interpretation and clinical management of patients with "Fixed" myocardial perfusion defects
}

\section{A call for quantifying endocardial-to-epicardial distribution of blood flow}

\author{
Vasken Dilsizian, MD, MASNC ${ }^{\mathrm{a}}$ \\ a Department of Diagnostic Radiology and Nuclear Medicine, University of Maryland School of \\ Medicine, Baltimore, MD
}

Received Dec 10, 2020; accepted Dec 10, 2020

doi: $10.1007 / \mathrm{s} 12350-020-02492-8$

In patients with exertional symptoms of chest pain or shortness of breath, myocardial perfusion imaging is commonly performed with exercise stress to induce coronary hyperemia and link the symptoms induced during exercise to the location, extent, and severity of abnormal perfusion patterns. During exercise stress, the increase in myocardial oxygen demand and limitation of oxygen supply create a supply-demand mismatch often resulting in cellular ischemia. However, a substantial proportion of patients are unable or incapable of exercising to an optimal level of exertion on the treadmill (defined as $>85 \%$ of the maximum predicted heart rate for age) to achieve coronary hyperemic responses. In such patients, pharmacologic stress testing can be used to induce coronary hyperemia. Unlike exercise stress, the perfusion defect induced with pharmacologic vasodilator stress may represent merely the heterogeneity in coronary flow reserve without inducing myocardial ischemia (with the exception of coronary steal). Typically, there is a modest increase in heart rate and decline in arterial pressure with vasodilator stress.

With the administration of vasodilators for pharmacologic stress, the resistance vessels in the area subtended by normal coronary arteries dilate, diminishing coronary resistance and resulting in an increase in coronary blood flow nearly 4-5 folds above normal. On the other hand,

Reprint requests: Vasken Dilsizian, MD, MASNC, Department of Diagnostic Radiology and Nuclear Medicine, University of Maryland School of Medicine, 22 South Greene Street, Baltimore, MD 21201; vdilsizian@umm.edu

J Nucl Cardiol 2021;28:723-8.

$1071-3581 / \$ 34.00$

Copyright (c) 2021 American Society of Nuclear Cardiology. because coronary resistance in myocardial regions supplied by a stenotic coronary artery are diminished at rest (i.e., coronary vasodilator reserve has been exhausted in order to sustain myocardial blood flow), only minor or no further reductions in coronary resistance can take place. Therefore, myocardial blood flow in stenotic coronary artery territory does not change with vasodilator stress or may even decrease slightly because of the peripheral vasodilation and drop in diastolic blood pressure characteristic of pharmacologic stress. The net result of these changes is heterogeneity in myocardial blood flow (increased in the normal territory and relatively unchanged in the territory supplied by a stenotic coronary artery), which will appear as a "fixed" defect on stress-rest myocardial perfusion imaging.

\section{DETECTION OF STRESS-INDUCED ISCHEMIA (“REVERSIBLE DEFECT") VERSUS INFARCTION (“FIXED DEFECT")}

In standard medical practice, exercise or pharmacologic stress myocardial perfusion images are compared with those acquired at rest to determine whether stress-induced perfusion defects reflect regional myocardial ischemia or prior infarction. Stress-induced perfusion abnormalities in regions that exhibit normal perfusion at rest are termed reversible perfusion defects, and such regions represent viable, ischemic tissue with blunted coronary blood flow reserve. On the other hand, perfusion abnormalities that are seen both on stress and rest myocardial perfusion images, are termed fixed or irreversible defects, which most often suggests an area of prior myocardial infarction, particularly if the defect is severe (with the exception of hibernation or repetitive 
stunning). When both viable myocardium and scarred myocardium are present, the defect may be characterized as mild-moderate fixed, which connotes nontransmural infarction, and can also be seen after percutaneous coronary intervention in the setting of acute coronary syndrome (Table 1). However, if the viable component of the admixture of viable and scarred myocardium is reversible (stress-induced ischemia), then the defect will appear partially reversible (a region of non-transmural infarction sub-served by significantly narrowed coronary artery).

What are the exceptions? A fixed perfusion defect with preserved wall motion can be attributed to soft tissue attenuation artifact, such as breast attenuation or inferior wall attenuation caused by the diaphragm, ascites, large pleural effusions, or by other abdominal visceral structures. However, in the presence of an abnormal wall motion, a fixed perfusion defect may also reflect hypoperfused myocardium at rest, subtended by a critically narrowed coronary artery, which will not change with stress. In the absence of prior history of myocardial infarction or pathologic Q-waves on the ECG, these patients may have viable, hibernating myocardium, and therefore, should undergo additional testing, such as FDG PET viability imaging (Table 1).

\section{CLINICAL AND REVASCULARIZATION CONSEQUENCES OF REVERSIBLE AND MILD TO MODERATELY REDUCED FIXED MYOCARDIAL PERFUSION DEFECTS}

The evaluation of myocardial ischemia and prior infarction by myocardial perfusion imaging studies has occupied a rather unique place in the management of patients with known or suspected coronary artery disease since the late 1970s. ${ }^{1,2}$ Substantial data now exist to indicate that in patients with coronary artery disease, impaired left ventricular function at rest is not necessarily an irreversible process. ${ }^{3}$ A viable myocardial region may have reduced contraction on the basis of repetitive stunning and/or hibernation and may improve after revascularization. Thus, a dysfunctional but viable myocardial region may have reduced (but not absent) blood flow at rest (termed hibernation), or transient reduction in blood flow after a period of ischemia (termed stunning). ${ }^{4,5}$ Regional left ventricular dysfunction arising from a transient period of myocardial ischemia (reversible defect) may improve after revascularization, while regional dysfunction arising from transmural or non-transmural myocardial infarction (fixed defect) may not recover function after revascularization.

It is now well established in the literature that while both reversible and mild-to-moderate fixed myocardial perfusion defects retain metabolically active, viable myocardium, stress-induced reversible perfusion defects will more accurately predict recovery of regional

Table 1. Scintigraphic patterns and interpretation of reversible and fixed myocardial perfusion defects

$\begin{array}{lll}\begin{array}{l}\text { Stress-Rest Paired } \\ \text { Interpretation }\end{array} & \begin{array}{c}\text { Further } \\ \text { Characterization }\end{array} & \text { Comments }\end{array}$

Reversible

Complete

Partial

Fixed

Mild-moderate

Severe

Extracardiac Artifact
Fixed with preserved regional wall motion
Ischemic and viable myocardium

Admixture of scarred and viable, ischemic myocardium (subtended by significantly narrowed coronary artery)

Admixture of scarred and viable, non-ischemic myocardium (subtended by non-significantly narrowed coronary artery, such as after percutaneous coronary intervention in the setting of acute coronary syndrome)

Transmural scar (in the absence of prior history of myocardial infarction or pathologic Q-waves on the ECG, these patients may have viable, hibernating myocardium, and should undergo additional viability imaging, such as with FDG PET)

These can be attributed to soft tissue attenuation artifact, such as breast attenuation or inferior wall attenuation caused by the diaphragm, ascites, large pleural effusions, or by other abdominal visceral structures 
function after revascularization (Figure 1). ${ }^{6,7}$ Although regions with mild-to-moderate fixed perfusion defects indeed retain viable myocardium, the mere presence of viable myocardium does not necessarily translate into recovery of function after revascularization. Even at a similar mass of viable myocardium (as reflected by the final radiotracer content and supported by histomorphological studies), the presence of inducible ischemia (a reversible defect) is associated with a higher likelihood of functional recovery when compared to mild-tomoderate fixed defect ( $83 \%$ vs $33 \%$, respectively; $P<$ $0.001){ }^{6}$

Because improvement in global left ventricular function after revascularization is associated with enhanced survival, it is clinically important to prospectively identify patients with functionally reversible myocardium from those who have predominantly scarred, non-recoverable myocardium. When taking into consideration regions with reversible perfusion defects (ischemia) and success of revascularization (re- examining regional perfusion or vessel patency after revascularization), the positive and negative predictive accuracies for recovery of function after revascularization is in the $80 \%-90 \%$ range. $^{6,8}$

\section{RECOVERY OF FUNCTION IS CLOSELY LINKED TO THE RATIO OF THE ADMIXTURE OF SCARRED AND VIABLE MYOCARDIUM WITHIN DYSFUNCTIONAL MYOCARDIAL REGIONS}

Histopathological studies have demonstrated the concept that tissue viability is a continuum. Given that uptake and retention of single photon emission computed tomography (SPECT) myocardial perfusion tracers, such as thallium, sestamibi, and tetrofosmin, reflect intact sarcolemmal Na-K ATPase or mitochondrial function, it would be anticipated that there would be a nearly linear relationship with the percentage of normal (viable) myocardium in dysfunctional

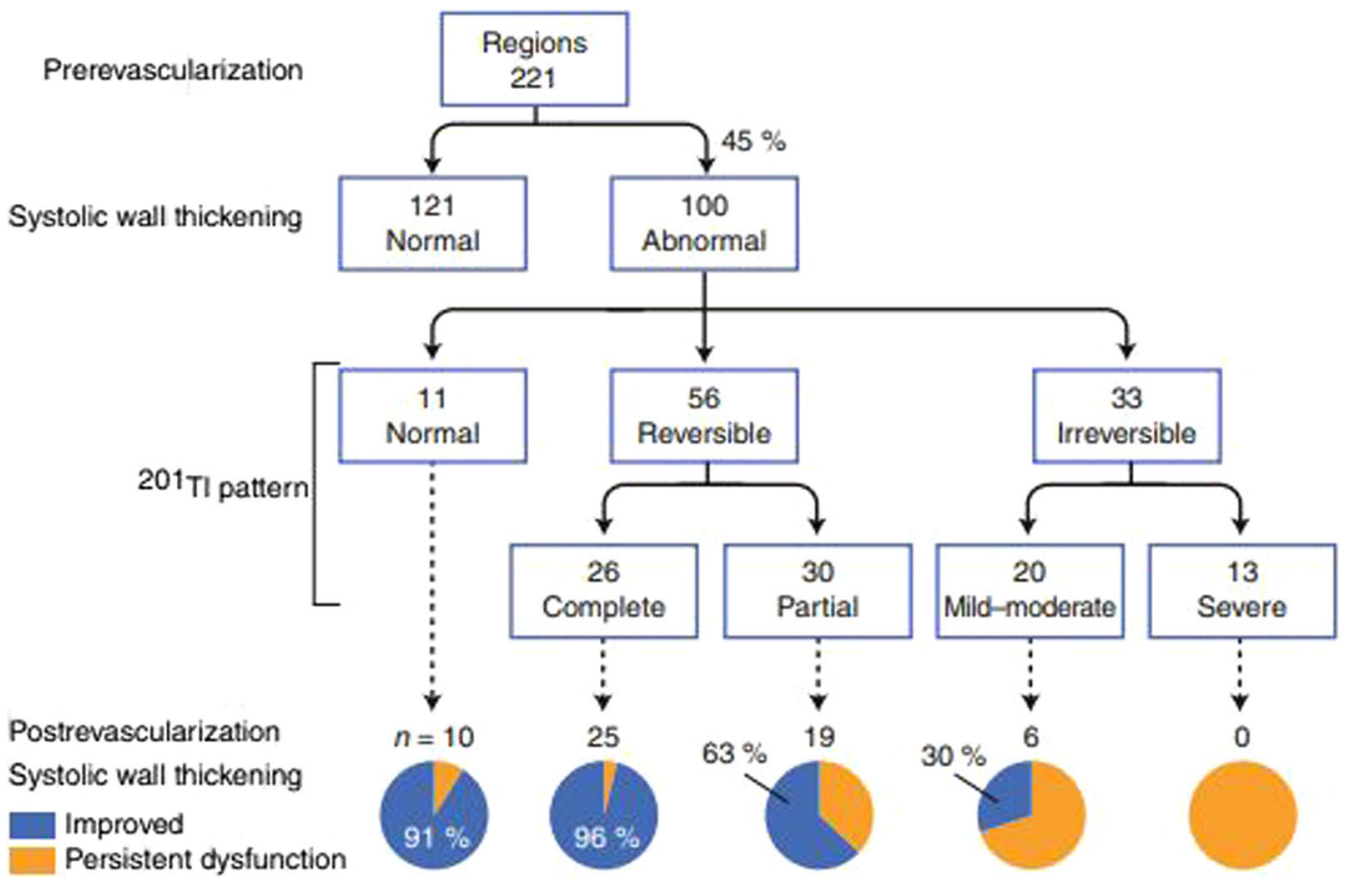

Figure 1. Flow diagram demonstrating post-revascularization functional outcome of asynergic regions in relation to pre-revascularization scintigraphic patterns of normal, reversible, partially reversible, mild-to-moderate irreversible, and severe irreversible defects using stress-redistributionreinjection thallium protocol. The probabilities of functional recovery after revascularization were over $90 \%$ in normal or completely reversible defects, $63 \%$ in partly reversible defects, $30 \%$ in mildto-moderate irreversible defects, and $0 \%$ in severe irreversible defects. Asynergic regions with reversible defects (complete or partial) were shown to be more likely to improve function after revascularization when compared with asynergic regions with mild-to-moderate irreversible defects (79 versus $30 \%$, respectively, $P<0.001$ ). Even at a similar mass of viable myocardial tissue (as reflected by the final radiotracer content), the presence of inducible ischemia (reversible defect) was associated with an increased likelihood of functional recovery (Modified from Kitsiou et al. ${ }^{6}$ ). 
regions. ${ }^{9,10}$ Indeed, several studies have shown a graded relationship between percent regional radiotracer uptake or percent transmural hyperenhancement on cardiac magnetic resonance and recovery of function after revascularization (Figure 2) ${ }^{11}$ Irrespective of the imaging modality applied, the data suggest that recovery of function after revascularization is a continuum and is coupled to the ratio of the admixture of scarred and viable myocardium within the dysfunctional regions. The extent of infarct size on cardiac magnetic resonance or percent thallium defect on SPECT correlated with decreasing likelihood of functional recovery after revascularization. The greater the extent of scarred myocardium, the lower the probability for recovery of function.

\section{QUANTITATIVE PET ABSOLUTE MYOCARDIAL BLOOD FLOW ASSESSMENT IN THE SUBENDOCARDIAL AND SUBEPICARDIAL COMPONENTS}

Radionuclide imaging lends itself well to an objective quantitative analysis because of its inherently digital nature. If the quantitative measures are highly reproducible, they may be better suited for assessing serial studies and/or for identifying differences in defect size and reversibility. ${ }^{12,13}$

One of the key advantages of positron emission tomography (PET) over SPECT is that it offers validated tracer kinetic models for quantifying absolute myocardial blood flow. ${ }^{14,15}$ PET permits reliable attenuation and scatter correction based on either external transmission sources or on CT images thereby enabling quantification of absolute myocardial blood flow in the heart, which represents the mean transmural myocardial blood flow. Unlike the high-resolution cardiac magnetic resonance, decreased perfusion limited to the subendocardium may not be detectable by mean transmural myocardial perfusion measured with conventional SPECT and PET cameras, unless the hypoperfusion extends further towards the subepicardium.

When compared to the subepicardium, the subendocardium is distinguished by differences in perfusion, function, loading and pressure constraints, rendering it more vulnerable for ischemia and injury in patients with coronary artery disease. The left ventricular intramural pressure is greatest near the endocardium and least near the epicardium. However, under normal conditions, this pressure gradient does not impair endocardial blood flow, because a greater blood flow to the endocardium during diastole compensates for the greater blood flow to the epicardium during systole, and the tone of the endocardial resistance vessels is less than the tone of the epicardial vessels.
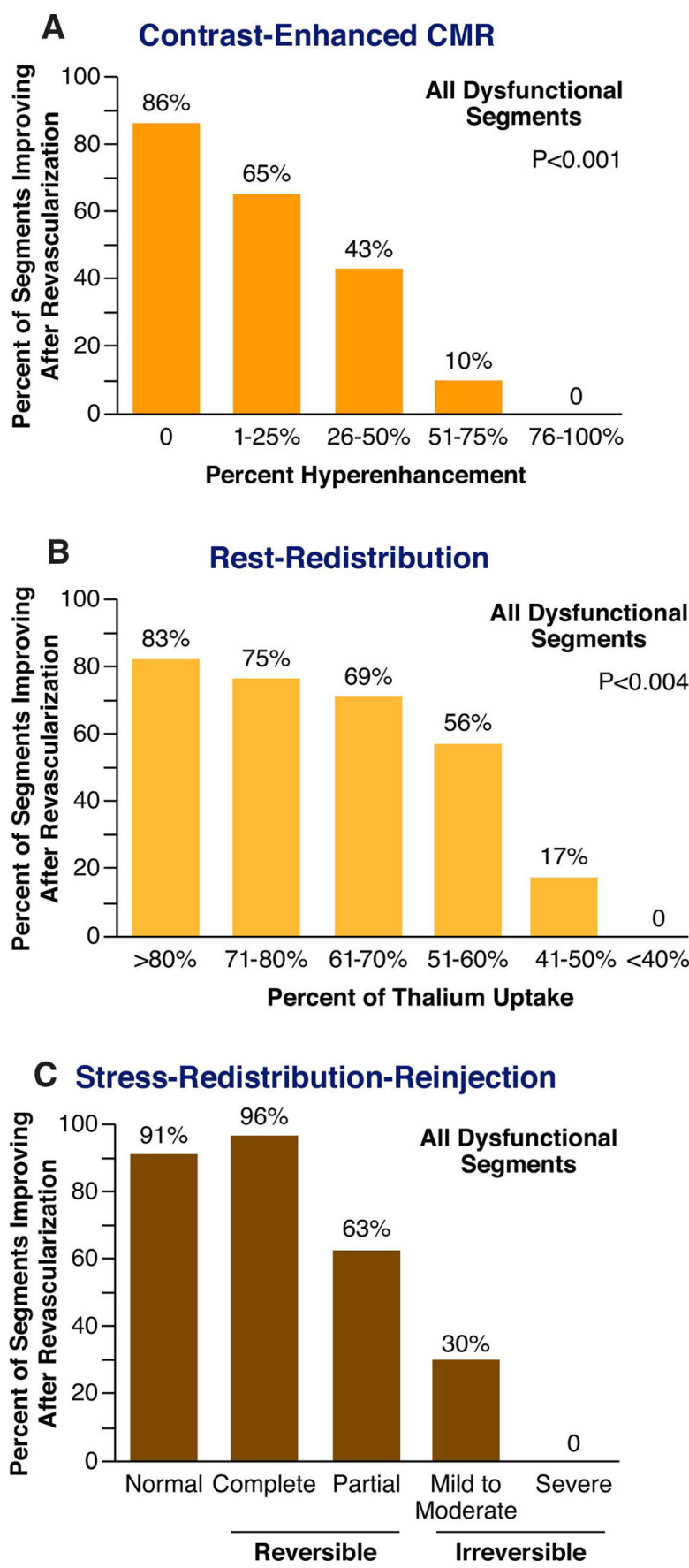

Figure 2. Irrespective of the imaging modality applied, recovery of function after revascularization is a continuum and is coupled to the ratio of viable to scarred myocardium within dysfunctional myocardial segments. Relationships between recovery of function after revascularization with contrast-enhanced cardiac magnetic resonance (A), rest-redistribution thallium (B), and stress-redistribution-reinjection $(\mathbf{C})$ imaging are shown. The extent of infarct size on cardiac magnetic resonance or percent thallium defect on SPECT correlated with decreasing likelihood of functional recovery after revascularization (Adapted from Dilsizian $\mathrm{V}^{11}$ ). 
At the present time, there are multitude of confounding factors and/or variables that may significantly impact the quantitative myocardial blood flow value. For example, averaging multiple segments and myocardial regions (apical, septal and anterior) in a single vascular territory (left anterior descending) underestimates visually discernible perfusion defects. When myocardial segments are between anatomical boundaries of major coronary artery vascular territories, such as the inferolateral region, it is often underestimated by global or vascular territory hyperemic myocardial blood flow values. Moreover, the quantified myocardial blood flow value is tightly dependent on image and data acquisition on PET scanner hardware (crystal sizes, ring diameter, axial extent of the ring), deadtime, saturation of one or more detector blocks at early time points, number of time frames and their duration, the inputs to kinetic modeling and type of kinetic model results applied, which can vary significantly amongst various centers, and the obtained values are not necessary interchangeable. ${ }^{16,17}$

In order to move the field towards dividing the PET transmural myocardial blood flow into subendocardial and subepicardial components, one has to be able to first reliably measure and reproduce blood flow measurements in smaller regions (5 regions) or segments (17 segments). Subendocardial and subepicardial myocardial blood flow has been previously calculated for each of the three coronary artery vascular territories. ${ }^{18}$ However, dividing the absolute myocardial perfusion parameter from a global index, to the 3 individual vascular territories, and further into 5 myocardial regions or 17 segments, indubitably increases the error of the measurement. To further split the transmural myocardial blood flow from an already small left ventricular myocardium region or segment into subendocardial and subepicardial components is a daunting task. The lower signal-to-noise ratio in these regions results in higher measurement variability, rendering the extracted quantifiable blood flow parameter unreliable for clinical decision making.

\section{FUTURE DIRECTIONS}

Advances in technological developments in instrumentation, software, radiopharmaceuticals, ${ }^{19}$ and processing of PET in the near future may result in optimization for quantifying endocardial-to-epicardial distribution of absolute myocardial blood flow. At the present time, respiratory and cardiac motion correction will help "freeze" the heart from motion, which will impact the overall spatial resolution of the imaging system and help to better demarcate the myocardial wall. However, the future would require improved PET spatial resolution in the range of $2-3 \mathrm{~mm}$ in order to reliably assess normal LV wall thickness $(6-10 \mathrm{~mm})$ and the possibility for dividing the myocardium into subendocardial and subepicardial layers. This may be achieved with improvement in coincidence time resolution (lower than $100 \mathrm{ps}$ ) through new PET detector designs, and some day even crossing the $10 \mathrm{ps}$, which will increase the performance of time-of-flight PET dramatically, resulting in a better signal-to-noise ratio of the activity measurement (e.g., F-18 Flurpiridaz), improving the reconstruction process and rendering quantifying the endocardial-to-epicardial distribution of myocardial blood flow achievable and attainable.

\section{References}

1. Pohost GM, Zir LM, Moore RH, McKusick KA, Guiney TE, Beller GA. Differentiation of transiently ischemic from infarcted myocardium by serial imaging after a single dose of thallium-201. Circulation 1977;55:294-302.

2. Akins CW, Pohost GM, Desanctis RW, Block PC. Selection of angina-free patients with severe left ventricular dysfunction for myocardial revascularization. Am J Cardiol 1980;46:695-700.

3. Dilsizian V, Bonow RO, Cannon RO, et al. The effect of coronary artery bypass grafting on left ventricular systolic function at rest: Evidence for preoperative subclinical myocardial ischemia. Am J Cardiol 1988;61:1248-54.

4. Berman M, Fischman AJ, Southern J, et al. Myocardial adaptation during and after sustained, demand-induced ischemia: Observations in closed-chest, domestic swine. Circulation 1996;94:755-62.

5. Gewirtz H, Dilsizian V. Myocardial viability: Survival mechanisms and molecular imaging targets in acute and chronic ischemia. Circ Res 2017;120:1197-212.

6. Kitsiou AN, Srinivasan G, Quyyumi AA, Summers RM, Bacharach SL, Dilsizian V. Stress-induced reversible and mild-tomoderate irreversible thallium defects: Are they equally accurate for predicting recovery of regional left ventricular function after revascularization? Circulation 1998;98:501-8

7. Dilsizian V, Freedman NMT, Bacharach SL, Perrone-Filardi P, Bonow RO. Regional thallium uptake in irreversible defects: Magnitude of change in thallium activity after reinjection distinguishes viable from nonviable myocardium. Circulation 1992;85:627-34.

8. Dilsizian V, Rocco TP, Freedman NM, Leon MB, Bonow RO. Enhanced detection of ischemic but viable myocardium by the reinjection of thallium after stress-redistribution imaging. $\mathrm{N}$ Engl $\mathrm{J}$ Med 1990;323:141-6.

9. Zimmermann R, Mall G, Rauch B, et al. Residual Tl-201 activity in irreversible defects as a marker of myocardial viability: Clinicopathological study. Circulation 1995;91:1016-21.

10. Dakik HA, Howell JF, Lawrie GM, et al. Assessment of myocardial viability with $99 \mathrm{mTc}$-sestamibi tomography before coronary bypass graft surgery: Correlation with histopathology and postoperative improvement in cardiac function. Circulation 1997;96:2892-8.

11. Dilsizian V. Cardiac magnetic resonance versus SPECT: Are all noninfarct myocardial regions created equal? J Nucl Cardiol 2007;14(1):9-14. 
12. Dilsizian V, Narula J. Qualitative and quantitative scrutiny by regulatory process: Is the truth subjective or objective? J Am Coll Cardiol Imaging 2009;2:1037-8.

13. Dilsizian V. Transition from SPECT to PET myocardial perfusion imaging: A desirable change in nuclear cardiology to approach perfection. J Nucl Cardiol 2016;23:337-8.

14. Dilsizian V, Bacharach SL, Beanlands SR, Bergmann SR, Delbeke D, Dorbala S, Gropler RJ, Knuuti J, Schelbert H, Travin M. ASNC Imaging Guidelines/SNMMI Procedure Standard for Positron Emission Tomography (PET) Nuclear Cardiology Procedures. J Nucl Cardiol 2016;23(5):1187-226.

15. Gewirtz H, Dilsizian V. Integration of quantitative PET absolute myocardial blood flow in the clinical management of coronary artery disease. Circulation 2016;133:2180-96.

16. Nesterov SV, Deshayes E, Sciagrà R, et al. Quantification of myocardial blood flow in absolute terms using (82)Rb PET imaging: the RUBY-10 Study. J Am Coll Cardiol Imaging 2014;7:1119-27.

17. Dilsizian V, Chandrashekhar Y, Narula J. Quantitative PET myocardial blood flow: "Trust, but verify". J Am Coll Cardiol Imaging 2017;10(5):609-10.

18. Danad I, Raijmakers PG, Harms HJ, et al. Impact of anatomical and functional severity of coronary atherosclerotic plaques on the transmural perfusion gradient: a $[15 \mathrm{O}] \mathrm{H}_{2} \mathrm{O}$ PET study. Eur Heart $\mathrm{J}$ 2014;35:2094-105.

19. Dilsizian V, Taillefer R. Journey in evolution of nuclear cardiology: Will there be another quantum leap with the F-18 labeled myocardial perfusion tracers? J Am Coll Cardiol Imaging 2012;5:1269-84.

Publisher's Note Springer Nature remains neutral with regard to jurisdictional claims in published maps and institutional affiliations. 